aqueous layer was recovered into a fresh tube and mixed with a half volume of $100 \%$ ethanol. Samples were applied to RNeasy Mini spin columns. RT-PCR, using Taqman low-density arrays, was used to determine gene expression.

Results The mean (SD) age and forced expiratory volume in 1 second of asthmatics was $36(13.4)$ years and $101.16(15.47) \%$ predicted respectively and for healthy volunteers was 36 (7.2) years and $92.16(17.43) \%$ predicted. MMP25 expression was significantly $(\mathrm{p}=0.04)$ higher and MMP15 expression was significantly $(\mathrm{p}=0.04)$ lower is asthmatics compared to healthy volunteers. ADAM28 was significantly $(\mathrm{p}=0.03)$ higher and ADAM17 and ADAMTS15 expression were significantly lower in asthma ( $\mathrm{p}=0.007 \& 0.008$ respectively). TIMP2 expression was significantly $(\mathrm{p}=0.007)$ lower in the asthma. There were no significant changes in expression of any of the metalloproteinases or their inhibitors after montelukast therapy.

Conclusion We have studied a wide range of known MMPs, ADAMs, ADAMTSs and inhibitors with a refined technique and successfully increased the yield from induced sputum samples. Significant differences were found between healthy volunteers and asthmatic patients for gene expression of some metalloproteinases/TIMPs. This technique could be used in the future when evaluating gene expression in asthma.

\section{P188 PREVALENCE OF SERUM EOSINOPHILIA AT TIME OF ADMISSION WITH AN EXACERBATION OF COPD}

DMG Halpin; Royal Devon \& Exeter Hospital, Exeter, UK

\subsection{6/thoraxjnl-2013-204457.340}

COPD is generally viewed as a disease driven by neutrophillic inflammation but up to $40 \%$ of COPD patients have an inflammatory pattern that includes elevated eosinophils (Saha, 2006) and there has been recent interest in the role of eosinophils in the aetiology and pathophysiology of exacerbations of COPD. Increased eosinophilic airway inflammation has been reported during exacerbations of COPD and peripheral eosinophils levels have been used as a surrogate to predict response to corticosteroid therapy. Treatment strategies with oral and inhaled steroids to reduce sputum eosinophils in COPD reduce exacerbation rates compared to

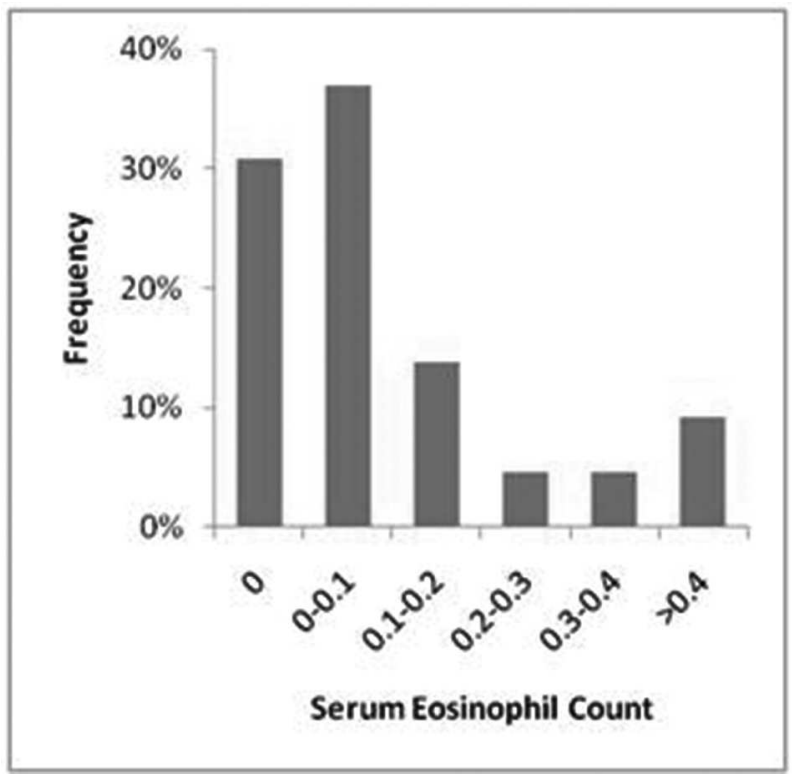

Abstract P188 Figure 1. a conventional care and there has been interest in using anti-eosinophil therapy to modify the clinical course of exacerbations.

Data were collected on 66 patients admitted with an acute exacerbation of COPD between Nov 2011 and Feb 2012 as part of an assessment of a discharge bundle. The mean age of the patients was 72 (range 49-91) and mean FEV1 33\% predicted.

Serum eosinophils were measured routinely in full blood counts performed at the time of admission. 20 patients (30\%) had no detectable eosinophils, and $6(9 \%)$ had raised eosinophil counts (normal range $\left.0.04-0.40 \times 10^{\wedge} 9 / 1\right)$. The median eosinophil count was 0.07 . One patient had an eosinophil count of 15.24 on admission, having previously had intermittently mildly elevated counts (up to 1.57) since at least 2000. Excluding this patient, the mean (SE) eosinophil count was $0.37(0.23)$. The distribution of serum eosinophil counts is shown in fig 1 .

In this group of patients, serum eosinophilia $(>0.4)$ was seen in only $9 \%$ of patients at the time of admission. This may have been affected by prior self management with oral steroids.

\section{REFERENCES}

1. Saha S, Brightling CE. Int J Chron Obstruct Pulmon Dis. 2006;1(1):39-47.

\section{P189 MITOCHONDRIAL DYSFUNCTION IN MUSCLE AND AIRWAY COMPARTMENTS IN COPD: PRELIMINARY FINDINGS}

G Haji, C Wiegman, M Patel, P Kemp, I Adcock, F Chung, M Polkey; Royal Brompton Hospital/Imperial College, London, England

\subsection{6/thoraxjnl-2013-204457.341}

Introduction \& Rationale Oxidative stress may underlie both pulmonary and non-pulmonary manifestations of COPD and may result from mitochondrial dysfunction. We hypothesised that if oxidative stress arose from the lung and 'spilled over' to cause non-pulmonary disease (e.g. skeletal muscle weakness) then greater evidence of mitochondrial dysfunction should be evident in the lung.

Objectives We measured mitochondrial function in endobronchial and skeletal muscle biopsies from COPD patients and healthy smokers matched for smoking history, age and sex.

Methods and Measurements We have so far biopsied 4 control smokers with normal $\mathrm{FEV}_{1}$ and $\mathrm{FEV}_{1} / \mathrm{FVC}$ ratio, and 4 GOLD II COPD patients out of a planned total of 40 . Bronchoscopy with endobronchial biopsies (EB) and percutaneous muscle biopsy of the vastus lateralis (VL) were obtained on the same day; additional phenotypic measurements included lung function, quadriceps strength and 6-minute walk (6MW) distance. Mitochondria were isolated and mitochondrial reactive oxygen species (ROS) and membrane potential (MP) were measured using MitoSOX Red and the carbocyanine dye JC-1 respectively and intracellular ROS determined by 2'-7'-dichlorofluorescin diacetate (DCF) staining. Results

\begin{tabular}{lllll}
\hline Mean \pm SEM & COPD & \multicolumn{3}{c}{ Controls } \\
Age (years) & $63 \pm 1$ & & $61 \pm 3$ & \\
FEV $_{1}$ (\% predicted) & $72 \pm 2$ & & $99 \pm 6$ & \\
QMVC (kg) & $33 \pm 8$ & & $49 \pm 6$ & \\
Smoking (pack-years) & $42 \pm 10$ & & $34 \pm 6$ & \\
$6 M W(m)$ & $490 \pm 47$ & & $635 \pm 50$ & \\
& VL & EB & VL & EB \\
Mitochondrial ROS (RFU) & $42196 \pm 9819$ & $244947 \pm 78170$ & $11983 \pm 1408$ & $93621 \pm 17981$ \\
Mitochondrial MP (units) & $4.6 \pm 1.0$ & $10.3 \pm 2.5$ & $4.9 \pm 1.3$ & $13.6 \pm 1.5$ \\
Intracellular ROS (RFU) & $22131 \pm 7195$ & $14820 \pm 5014$ & $727 \pm 297$ & $752 \pm 165$ \\
\hline
\end{tabular}

